KANNO, T.

KODAI MATH. SEM. REP

25 (1973), 446-448

\title{
AUTOMORPHISMS OF THE GALOIS GROUP OF THE ALGEBRAIC CLOSURE OF THE RATIONAL NUMBER FIELD
}

\author{
By Tsuneo Kanno
}

For any Galois extension $K / k$, let $\mathrm{Gal}(K / k)$ be the topological Galois group of $K / k$. Let $Q$ be the rational number field and let $\bar{Q}$ be the algebraic closure of $Q$. For algebraic number field $K$, let $\tilde{K}$ be the composite of all solvable extensions of $K$, and put $G_{K}=\operatorname{Gal}(\bar{Q} \mid K)$ and $\tilde{G}_{K}=\mathrm{Gal}(\tilde{K} \mid K)$.

In [1] and [2] Neukirch proved that for algebraic number fields $K_{1}$ and $K_{2}$ which are finite Galois extensions of $Q, G_{K_{1}} \simeq G_{K_{2}}$ (or $\tilde{G}_{K_{1}} \simeq \tilde{G}_{K_{2}}$ ) implies $K_{1}=K_{2}$, and in [2] he gave a conjecture to the effect that any automorphism of $G_{Q}$ (or $\tilde{G}_{Q}$ ) is inner. By his theorem we have that $\sigma\left(G_{K}\right)=G_{K}$, for any automorphism $\sigma$ of $G_{Q}$ (or $\tilde{G}_{Q}$ ) and for any number field $K$ which is a finite Galois (or solvable, res.) extension of $Q$; thus we have that $\sigma$ induces an automorphism $\sigma_{K}$ of $\mathrm{Gal}(K / Q)$. If by $\operatorname{Aut}_{0}(\mathrm{Gal}(K / Q))$ we denote the subgroup of the automorphism group $\operatorname{Aut}(\mathrm{Gal}(K / Q))$ of $\mathrm{Gal}(K / Q)$, consisting of those elements which leave any normal subgroup of $\mathrm{Gal}(K / Q)$ invariant, we have that the mapping $\sigma \mapsto\left(\sigma_{K}\right)_{K}$ gives a canonical isomorphism of the automorphism group Aut $\left(G_{Q}\right)$ (or Aut $\left(\tilde{G}_{Q}\right)$ ) onto the projective limit $\lim \operatorname{Aut}_{0}(\operatorname{Gal}(K / Q))$, where $K$ runs among the number fields which are finite Galois

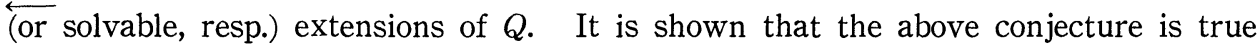
if and only if any $\sigma \varepsilon$ Aut $\left(G_{Q}\right)$ (or Aut $\left(\tilde{G}_{Q}\right)$ ) induces an inner automorphism $\sigma_{K}$ for any finite Galois (or solvable, resp.) extension $K$ of $Q$.

As Neukirch pointed out in [2], it is natural to consider some kind of group extensions to solve this problem. In this note we shall show that $\sigma_{K}$ is inner for a certain class of finite Galois (or solvable) extensions $K$ of $Q$, at least for any finite abelian extension $K$ of $Q$.

Let $G=\left\{g, g_{1}, g_{2}, \cdots\right\}$ be a finite group and let $A=\left\{a, a_{1}, a_{2}, \cdots\right\}$ be a finite abelian group. Let $\theta$ be a homomorphism of $G$ into the automorphism group $(A)$ of $A$ and let

$$
G \times A \ni(g, a) \longmapsto g \circ a=\theta(g)(a) \in A
$$

be the operation of $G$ on $A$ by $\theta$. Let $\hat{G}$ be the semidirect product $A \times{ }_{\theta} G$ of $A$ and $G$ by $\theta$ : i.e. $\hat{G}$ is the group which is $A \times G$ as set and in which the group operation is given by

Recelved November 30, 1972. 


$$
\left(a_{1}, g_{1}\right)\left(a_{2}, g_{2}\right)=\left(a_{1} \cdot g_{1} \circ a_{2}, g_{1} g_{2}\right) .
$$

For any automorphism $\sigma$ of $\hat{G}$, let $\sigma_{A}$ and $\sigma_{G}$ be the mappings: $\hat{G} \rightarrow A$ and $\hat{G} \rightarrow G$, respectively, defined by

$$
\sigma(a, g)=\left(\sigma_{A}(a, g), \sigma_{G}(a, g)\right) .
$$

Applying $\sigma$ on (1), we have

$$
\begin{aligned}
& \sigma_{A}\left(a_{1} \cdot g_{1} \circ a_{2}, g_{1} g_{2}\right)=\sigma_{A}\left(a_{1}, g_{1}\right) \cdot \sigma_{G}\left(a_{1}, g_{1}\right) \circ \sigma_{A}\left(a_{2}, g_{2}\right), \\
& \sigma_{G}\left(a_{1} \cdot g_{1} \circ a_{2}, g_{1} g_{2}\right)=\sigma_{G}\left(a_{1}, g_{1}\right) \sigma_{G}\left(a_{2}, g_{2}\right) .
\end{aligned}
$$

From (3) it follows that $\sigma_{G}$ is a homomorphism of $\hat{G}$ into $G$.

Suppose that $\sigma$ induces an automorphism of $G /(A \times e)$; i.e.

$$
\sigma_{G}(a, e)=e
$$

where $e$ is the identity element of the corresponding group. Substituting $g_{1}=g_{2}=e$ in (2), we have that the restriction $\sigma_{A}$ to $A \times e$ is an endomorphism of $A \times e$, which is denoted by the same $\sigma_{A}$. Since $\sigma$ is injective and $A$ is finite, $\sigma_{A}$ is an automorphism of $A \times e$.

Substituting $g_{1}=e, a_{2}=e$ in (2) and using (4), we have

$$
\sigma_{A}(a, g)=\sigma_{A}(a, e) \sigma_{A}(e, g)
$$

Substituting $a_{1}=e, g_{2}=e$ in (2), we have

$$
\sigma_{A}(g \circ a, g)=\sigma_{A}(e, g) \cdot \sigma_{G}(e, g) \circ \sigma_{A}(a, e) .
$$

Since $A$ is abelian, from (5) and (6) it follows

$$
\sigma_{A}(g \circ a, e)=\sigma_{G}(e, g) \circ \sigma_{A}(a, e) .
$$

On the other hand, sustituting $g_{1}=e$ in (3) and using (4) we have

$$
\sigma_{G}\left(a_{1} a_{2}, g\right)=\sigma_{G}\left(a_{2}, g\right) .
$$

Hence

$$
\sigma_{G}(a, g)=\sigma_{G}(e, g)
$$

and the mapping $g \mid \rightarrow \sigma_{G}(e, g)$ is the automorphism $f$ of $G$ induced by $\sigma$.

Suppose that $\theta$ is an isomorphism of $G$ onto Aut $(A)$, then there exists $x \in G$ such that $\theta(x)=\sigma_{A}$ and from (7) it follows

$$
\theta(x) \theta(g)=\theta(f(g)) \theta(x)
$$

Hence we have $f(g)=x g x^{-1}$.

Now we have

LEMMA. Let $\theta$ be an isomorphism of a finite group $G$ onto the automorphism 
group of a finite abelian group $A$, and let $\hat{G}=A \times{ }_{\theta} G$ be the semidirect product of $A$ and $G$ by $\theta$, then any automorphism $\sigma$ of $\hat{G}$ such that $\sigma(A \times e) \subset A \times e$ induces an inner automorphism of $G$.

ExAmple. For the cyclic group $A$ of order $m$ and the unit group $G=(Z / m Z)$ * of the ring $Z / m Z$, where $Z$ is the integer ring, we have an isomorphism $\theta: G$ $\simeq \operatorname{Aut}(A)$.

Theorem. Let $K$ be a finite Galois (or solvable) extension of $Q$ such that there exists a splitting extension

$$
1 \longrightarrow N \longrightarrow \operatorname{Aut}(A) \longrightarrow \operatorname{Gal}(K / Q) \longrightarrow 1
$$

where $N$ is a finite nilpotent group, $A$ is a finite abelian group and Aut $(A)$ is the automorphism group of $A$. Then any automorphism of $G_{Q}$ (or $\tilde{G}_{Q}$, res.) induces an inner automorphism of $\mathrm{Gal}(K / Q)$.

Proof. The Safarevic imbedding theorem [3] shows that the extension $K / Q$ is imbedded in a finite Galois extension $E / Q$ such that $\operatorname{Gal}(E / Q)=$ Aut $(A)$ and $\operatorname{Gal}(E / K)=N$. Again, the Šafarevič theorem and the above lemma show that any automorphism of $G_{Q}$ (or $\tilde{G}_{Q}$ ) induces an inner automorphism of $\mathrm{Gal}(E / Q)$ and induces an inner automorphism of $\mathrm{Gal}(K / Q)$ also.

Corollary. Any automorpism of $G_{Q}$ or $\tilde{G}_{Q}$ induces the identity automorphism of the Galois group of any finite abelian extension of $Q$.

Proof. Since any finite abelian extension of $Q$ is contained in some cyclotomic field, the theorem and the above example give the corollary.

\section{BIBLIOGRAPHY}

[1] Neukirch, J., Kennzeichnung der $p$-adischen und der endlichen algebraischen Zahlkörper. Inventıones math. 6 (1969) 296-314.

[2] Neukirch, J., Kennzeichnung der endlich-algebraischen Zahlkörper durch die Galoısgruppe der maximal auflösbaren Erweiterung. J. Reıne Angew. Math. 238 (1969), 135-147.

[3] Šafarevič, I. R., On the problem of imbedding fields. Izv. Akad. Nauk SSSR Ser. Mat. 18 (1954), 389-418; Amer. Math. Soc. Translations 4.

Department of Mathematics,

Tokyo Institute of Technology. 